\title{
The Effects of Antidepressants on Neuropeptide $Y$ in Patients with Depression and Anxiety
}

Authors

Affiliations

\author{
S. Ozsoy ${ }^{1}$, O. Olguner Eker ${ }^{2}$, U. Abdulrezzak ${ }^{3}$
}

${ }^{1}$ Department of Psychiatry, Erciyes University School of Medicine, Kayseri,Turkey ${ }^{2}$ Department of Psychiatry, Kayseri Education and Research Hospital, Kayseri, Turkey ${ }^{3}$ Department of Nuclear Medicine, Erciyes University School of Medicine, Kayseri, Turkey

\author{
Key words \\ depression \\ anxiety \\ - antidepressant \\ neuropeptide y
}

received 03.06.2015

revised $\quad 15.10 .2015$

accepted 20.10.2015

Bibliography

DOI http://dx.doi.org/

10.1055/s-0035-1565241

Published online:

January 20, 2016

Pharmacopsychiatry 2016;

49: 26-31

(c) Georg Thieme Verlag KG

Stuttgart - New York

ISSN 0176-3679

\section{Correspondence}

\section{S. Ozsoy}

Department of Psychiatry

Erciyes University School of Medicine

38039-Kayseri/Turkey

sozsoy@erciyes.edu.tr

\section{Abstract}

$\nabla$

Introduction: This study aimed to investigate the neuropeptide Y (NPY) levels in patients with anxiety and depression and also the effects of antidepressants on this neuropeptide.

Materials and Methods: The study included 40 outpatients who presented with depressive and anxiety symptoms, and 32 healthy controls. The patients received antidepressant treatment for 6 months. Serum levels of neuropeptide Y were measured before treatment in 40 patients, after 8 weeks of treatment in 32 patients, after 6 months in 10 patients, and once in the controls.

\section{Introduction}

$\nabla$

Neuropeptide Y (NPY) is a neuropeptide widely distributed in the central nervous system. NPY plays a regulatory role in several areas such as feeding, body weight, blood pressure, sleep, cognition, and emotion [1]. Most receptors of NPY are densely expressed in the cortex, hippocampus, and amygdala, which are brain regions associated with emotion regulation and stress response [2]. Peripherally, NPY is expressed in the sympathetic nerves and adrenal gland. Peripheral and central NPY regulates the release and activity of norepinephrine (NE) [3]. Preclinical data have suggested that NPY is secreted in response to stress, and intracerebroventricular administration of NPY shows anxiolytic and antidepressant-like effects in animals [4]. Clinical data have also proposed that NPY is involved in the pathophysiology and treatment of stressrelated disorders such as depression and anxiety [5]. Preliminary reports have provided evidence for the potential role of NPY and/or NPY receptor agonist/antagonists as a novel antidepressant [6]. The antidepressant and anxiolytic effect of NPY may be related to its antagonistic effect on the
Results: Serum NPY levels were lower in the patients than in the controls. NPY levels were increased and normalized by antidepressant treatment. While there was no change in NPY levels in the patients using fluoxetine and sertraline for 8 weeks, an increase was found in patients using escitalopram and venlafaxine. Serum NPY levels were increased by treatment for 8 weeks in the patients with depression, but not in the patients with anxiety.

Discussion: The findings suggest that NPY may be related to pathophysiology in depression and anxiety, and antidepressants influence NPY levels.

hypothalamic-pituitary-adrenal (HPA) axis [7]. It has been suggested that NPY is reduced in depression as it is associated with HPA axis hyperactivity. Many clinical studies have revealed low levels of cerebrospinal fluid (CSF) and plasma NPY in depressive patients [8-11]. However, some studies have observed an increase [12] or no significant change [13] in plasma NPY levels in depression. Contrary to previous findings, some recent studies reported high levels of CSF NPY in depressive patients [14]. Low CSF and plasma NPY levels have also been reported in post-traumatic stress disorder $[15,16]$. A few earlier studies that investigated plasma NPY levels found contradictory results in patients with anxiety disorder, such as panic disorder $[17,18]$. These reports demonstrated the role of NPY in stress and anxiety in addition to depression.

Although some studies have suggested an increase in NPY levels with antidepressant treatment as consistent with low NPY levels in depression, the results of studies about the effect of antidepressant treatment on NPY are controversial. While Nikisch et al. (2005) reported increased CSF NPY-like immunoreactivity after 4 weeks of treatment with the SSRI citalopram 
[19]. Martinez et al. (2012) reported no change in CSF NPY with venlafaxine [14]. Another study demonstrated decreased levels of CSF NPY as an effect of long-term antidepressant treatment in suicide attempters [20]. There is no study investigating peripheral NPY activity in relation to the effects of antidepressants.

Although central NPY activity seems to be more related to psychopathology, plasma NPY levels are commonly used to investigate NPY activity in stress and mental health research in humans. However, the relationship between central and peripheral NPY is poorly understood. Nevertheless, it has been suggested that peripheral NPY is a marker of stress-resilience [21] and is also related to psychopathology in stress-related disorders [5]. It seems that both central and peripheral NPY may have a role in the pathophysiology of stress-related disorders such as depression and anxiety. Additionally, antidepressant treatment may have different effects on NPY according to the type of antidepressant agent used or treatment duration. However, these issues have still not been clarified. Therefore, the present study was carried out to test the hypothesis of reduced peripheral NPY-ergic activity in depression and anxiety and focused on the effect of frequently used antidepressant agents on serum NPY levels in patients with depression and anxiety.

\section{Materials and Methods}

$\nabla$

\section{Subjects}

Forty inpatients (5 men and 35 women, mean age \pm SD: $32.03 \pm 9.69$, age range: $20-49$ ) were recruited for the study. The patients were selected consecutively from the outpatient population who applied to the Psychiatric Clinic of Erciyes University Medical School because of symptoms of depression and anxiety. The patients met the DSM-IV criteria for major depressive disorder (MDD) $(n=8)$, generalized anxiety disorder (GAD) $(n=8)$ or adjustment disorder $(n=24)$. Although epidemiological researches are rare, adjustment disorders and generalized anxiety disorder (GAD) are prevalent disorders in outpatient psychiatric populations in our country [22-24]. Therefore, adjustment disorder and GAD were selected in addition to major depression. The DSM-IV diagnoses were determined via clinical interviews. The patients had been psychotropic drug-free for at least 6 months.

32 physically and mentally healthy controls ( 5 men and 27 women, mean age \pm SD: $31.50 \pm 7.30$, age range: $20-49$ ) were recruited from among volunteer hospital staff.

The exclusion criteria were as follows: any comorbid DSM-IV disorder including psychotic disorder, bipolar disorder, eating disorder, and alcohol-drug use disorder (except smoking) for patients, any psychiatric disorder for controls, and medical illness (e.g., metabolic, endocrine) and use of hormonal medication for all subjects. Medical disorders were excluded through history, clinical examination, and evaluating the results of laboratory tests. All patients and controls underwent routine biochemical and hematological laboratory tests. None of the subjects was in a weight reducing program.

The study was approved by the local Ethics Committee of Erciyes University. Written informed consent was obtained from all patients and controls after the study had been fully explained to them.

\section{Procedure}

The patients were examined at 3 time points (pre-treatment, and at 8 weeks and 6 months after initiation of antidepressant treatment). The severity of clinical symptomatology was assessed using the Hamilton Depression Rating Scale (HAM-D) for depression and the Hamilton Anxiety Rating Scale (HAM-A) for anxiety. Antidepressant drugs were started immediately after pre-treatment examinations in the patients. The patients continued to use the same antidepressant agent throughout the study until month 6 . Those patients who needed to change or augment treatment were excluded for later assessments. 32 patients were able to complete 8 weeks. 9 of them were treated with sertraline (50 mg daily), 9 with escitalopram (10-20 mg daily), 7 with fluoxetine ( $20 \mathrm{mg}$ daily), and 7 with venlafaxine (75-150 $\mathrm{mg}$ daily) in the psychiatric outpatient clinic. The patients did not receive any additional drug including benzodiazepines or non-drug therapies such as psychotherapy. Only 10 patients were able to complete the study protocol for 6 months. The diagnostic compositions of these 10 patients were 1 patient with MDD, 1 patient with GAD and 8 patients with adjustment disorder.

Serum levels of NPY were measured before the initiation of the treatment, and at 8 weeks and 6 months after initiation of antidepressant treatment in the patients, and only once in the control subjects. Blood samples for NPY measurement were taken with a catheter inserted into the antecubital vein at 08:00-09:00 in the morning after an overnight fast. Separated serum was stored at $-70^{\circ} \mathrm{C}$ until analyzed. Serum NPY levels were determined by radioimmunoassay kit (Phoenix Pharmaceuticals, Inc., USA). The assay range was $10-1280 \mathrm{pg} / \mathrm{ml}$. All samples were run in a single assay. The sensitivity was $26 \mathrm{pg} / \mathrm{ml}$ and the intraassay coefficient of variation was $6.7 \%$.

\section{Statistical analysis}

The distributions of all variables were checked with the ShapiroWilk test. The patients and controls were compared with respect

\begin{tabular}{|c|c|c|c|c|c|}
\hline \multicolumn{4}{|c|}{ Patients } & \multicolumn{2}{|c|}{ Comparisons } \\
\hline & $\begin{array}{l}\text { Pre-treatment } \\
n=40\end{array}$ & $\begin{array}{l}8 \text { weeks after- } \\
\text { treatment } \\
n=32\end{array}$ & $\begin{array}{l}\text { Controls } \\
n=32\end{array}$ & $\begin{array}{l}\text { Pre-treatment- } \\
\text { control }\end{array}$ & $\begin{array}{l}8 \text { weeks } \\
\text { after treatment- } \\
\text { control }\end{array}$ \\
\hline $\begin{array}{l}\text { NPY }(p g / m l) \\
(\text { mean } \pm S D)\end{array}$ & $68.9 \pm 28.74^{*}$ & $79.5 \pm 33.85^{*}$ & $98.56 \pm 35.06$ & $\begin{array}{l}t=3.946 \\
p<0.001\end{array}$ & $\begin{array}{l}t=2.213 \\
p=0.031\end{array}$ \\
\hline $\begin{array}{l}\text { NPY (corrected } \\
\left.\text { mean }{ }^{* *} \pm S E\right)\end{array}$ & $69.81 \pm 5.11^{*}$ & $80.95 \pm 6.26$ & $97.42 \pm 5.74$ & $\begin{array}{l}F=12.382 \\
\mathbf{p}=\mathbf{0 . 0 0 1}\end{array}$ & $\begin{array}{l}F=3.173 \\
p=0.080\end{array}$ \\
\hline
\end{tabular}

Table 1 Serum NPY levels of patients before and after treatment and controls. 
Table 2 Demographic and clinical characteristics of the subjects.

\begin{tabular}{|c|c|c|c|c|c|}
\hline & \multicolumn{2}{|c|}{ Patients } & \multirow[b]{2}{*}{$\begin{array}{l}\text { Controls } \\
\text { Median(IR) } \\
\mathrm{n}=32\end{array}$} & \multicolumn{2}{|c|}{ Comparisons } \\
\hline & $\begin{array}{l}\text { Pre-treatment } \\
\text { Median(IR) } \\
\mathrm{n}=\mathbf{4 0}\end{array}$ & $\begin{array}{l}\text { Post-treatment } \\
\text { Median(IR) } \\
n=32\end{array}$ & & $\begin{array}{l}\text { Pre-treatment- } \\
\text { control }\end{array}$ & $\begin{array}{l}\text { Post-treatment- } \\
\text { control }\end{array}$ \\
\hline Age & $31(15)$ & - & $29.5(9)$ & $\begin{array}{l}Z=0.102 \\
p=0.919\end{array}$ & - \\
\hline Women/men & $35 / 5$ & - & $27 / 5$ & $\begin{array}{l}X^{2}=0.145 \\
p=0.703\end{array}$ & - \\
\hline BMI & $26.7(6.98)^{*}$ & $26.85(6.15)^{*}$ & $23(3.52)$ & $\begin{array}{l}Z=2.431 \\
p=\mathbf{0 . 0 1 5}\end{array}$ & $\begin{array}{l}Z=2.652 \\
p=\mathbf{0 . 0 0 8}\end{array}$ \\
\hline Total duration of illness (year) & $2(4)$ & - & - & - & - \\
\hline Duration of present episode (month) & $5.5(11)$ & - & - & - & - \\
\hline Number of episodes & $2(1)$ & - & - & - & - \\
\hline HAM-D & & & & - & - \\
\hline MDD & $26(5)(n=8)$ & $13(2)(n=6)$ & & - & - \\
\hline GAD & $23.5(6.75)(n=8)$ & $5(14.5)(n=5)$ & & - & - \\
\hline Adjustment disorder & $18(6.75)(n=24)$ & $8(6.5)(n=21)$ & & $Z=7.307$ & $Z=6.302$ \\
\hline All patients & $20.5(8) *(n=40)$ & $11(7.75) *(n=40)$ & $0(2)$ & $\mathrm{p}<0.001$ & $\mathrm{p}<0.001$ \\
\hline HAM-A & & & & - & - \\
\hline MDD & $33(9)(n=8)$ & $15(18.25)(n=6)$ & & - & - \\
\hline GAD & $41.5(8)(n=8)$ & $27(24.5)(n=5)$ & & - & - \\
\hline Adjustment disorder & $23.5(11.75)(n=24)$ & $10(16)(n=21)$ & & $Z=7.266$ & $Z=5.895$ \\
\hline All patients & $28.5(17) *(n=40)$ & $11.5(18.75) *(n=32)$ & 3.5 (2.75) & $p<0.001$ & $\mathrm{p}<0.001$ \\
\hline
\end{tabular}

BMI: Body mass index, GAD: Generalized anxiety disorder, HAM-A: Hamilton Anxiety Rating Scale, HAM-D: Hamilton Depression Rating Scale, MDD: Major depressive disorder, IR: Inter-quartile range

*: Higher than those of the controls

to sex by using the chi-square test. The significance of differences between the demographic and clinical characteristics of the patients and controls was assessed with the Mann-Whitney $\mathrm{U}$ test because of the abnormal distribution. To compare the NPY levels of the patients and controls, the independent samples t-test was used. To control the confounding effect of BMI, the univariate-ANCOVA test was also performed for the latter comparison by taking BMI as covariate. Estimated marginal means for NPY values were calculated by taking BMI as covariate in the univariate-ANCOVA test and were given in $\bullet$ Table $\mathbf{1}$ as corrected means. The paired samples t-test was used for the comparison of the NPY levels of patients before and after 8 weeks of treatment. Serum NPY levels at 3 time points (pre-treatment, and at 8 weeks and 6 months after initiation of treatment) were compared with the nonparametric Friedman test because of the small sample size $(n=10)$. The Wilcoxon test was used for the analyses of subgroups according to type of illness and antidepressant agent. The Spearman correlation test was performed to investigate the relationships between NPY levels and clinical and demographical variables in the patients and controls separately.

\section{Results}

$\nabla$

The patients and controls did not differ significantly with regard to gender and age. The BMI of the patients was higher than that of the controls. The depression and anxiety scores of the patients were higher than those of the controls before and after treatment ( $\bullet$ Table 2 ).

The serum NPY levels of the patients were lower than those of the controls both before and after 8 weeks of treatment. When corrected means were compared by controlling the effect of BMI with

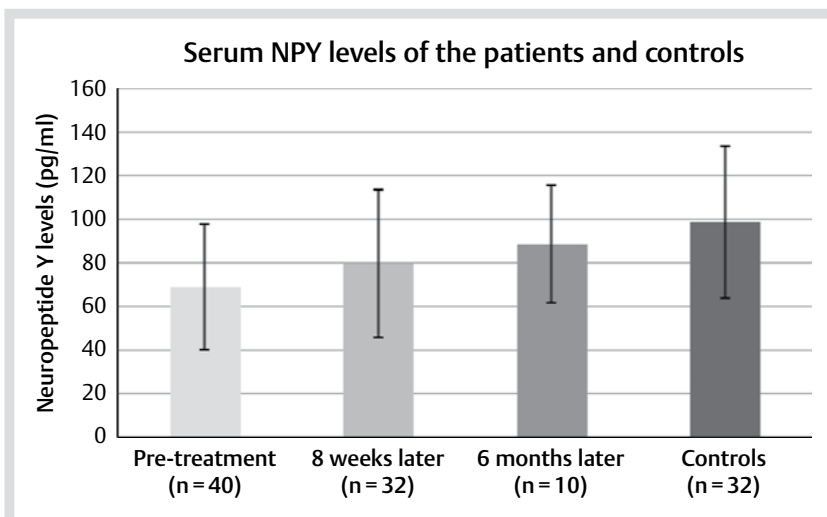

Fig. 1 Serum neuropeptide $Y$ levels (mean \pm standard deviation) of the controls and patients pre-treatment as well as 8 weeks and 6 months after initiation of antidepressant treatment.

ANCOVA, the serum NPY levels of the patients were lower than those of the controls before treatment. However, after 8 weeks of treatment, they were similar to those of the controls ( $\bullet$ Table 1 ). The comparison of serum NPY levels before and after 8 weeks of treatment was carried out with paired samples t-test for 32 patients. The NPY levels of patients after 8 weeks of treatment $(79.5 \pm 33.85)(n=32)$ were higher than the pre-treatment levels $(65.12 \pm 24.88)(n=32)(t=2.052 \mathrm{p}=0.049)$. Serum NPY levels at 3 time points (pre-, after 8 weeks of treatment, and 6 months later) could only be compared in 10 patients and there was a significant difference among NPY levels at these time points. While there was no significant difference between NPY levels pre-treatment (mean \pm SD: $61.40 \pm 27.35$ ) and after 8 weeks of treatment (mean \pm SD: $70.70 \pm 31.74)(\mathrm{p}=0.517)$, serum NPY levels after 6 months (mean \pm SD: $88.60 \pm 26.78$ ) were higher than 
both those at pre-treatment $(\mathrm{p}=0.009)$ and after 8 weeks of treatment $(p=0.037)$. In $\odot$ Fig. 1 , the serum NPY levels at 3 different time points are shown for all patients at those times.

While the NPY levels of the patients were not changed by 8 weeks of treatment in patients receiving sertraline and fluoxetine, they were increased by 8 weeks of treatment in patients receiving escitalopram $(\mathrm{Z}=2.073, \mathrm{p}=0.038)$ and venlafaxine $(\mathrm{Z}=2.197, \mathrm{p}=0.028)$. In the patients diagnosed with major depressive disorder, NPY levels $(Z=1.992, p=0.046)$ and BMI $(\mathrm{Z}=2.207, \mathrm{p}=0.027)$ were increased after 8 weeks of treatment. No change in NPY levels of the other diagnostic groups with 8 weeks of treatment was found. When the NPY levels of only women were compared, increased NPY levels were found after 8 weeks of treatment compared to those pre-treatment $(\mathrm{Z}=2.427$, $\mathrm{p}=0.015)(\otimes$ Table 3$)$.

\section{Discussion}

The main finding of the present study is that the serum NPY levels in the patients were lower than those in the controls. The finding is in accordance with previous studies that have reported decreased central and peripheral NPY levels in patients with depressive and anxiety disorders $[8,10,11,15,16]$. NPY deficits in various regions of the brain have been associated with sympathetic overactivity, reduced stress-resilience, impaired stress response, and anxiety [25]. The finding of the present study together with these reports might suggest reduced central and peripheral NPY-ergic activity in stress-related disorders. Reduced NPY-ergic activity in stress-related disorders such as depression may be related to the antagonistic action of NPY on the HPA axis and stress response system. Available data have shown that NPY administration might reverse HPA axis hyperactivity. It has been suggested that NPY suppresses HPA axis activity by reducing the corticotropin-releasing hormone (CRH), adrenocorticotrophic hormone (ACTH), and cortisol [7]. Decreased NPY levels are probably associated with increased HPA activity in depression. One can consider that some depressive symptoms such as loss of weight and appetite might be related to reduced NPY levels. NPY has an orexigenic action and stimulates food intake and decreases energy expenditure [26].
Low NPY levels may be related to loss of appetite which is a symptom of depression and anxiety.

In the present study, we found that NPY levels increased and were close to those of the controls. Increasing NPY levels after antidepressant treatment are also consistent with an earlier study [19]. The normalization of NPY levels may be related to normalization of HPA axis activity after treatment in depressive patients. It is known that HPA axis activity is normalized by treatment for depression [27]. Additionally, normalization of NPY levels after treatment may also be a result of improvement of symptoms such as lack of appetite. However, NPY levels may also be associated with anxiety symptoms. NPY may play a role in regulating anxiety, possibly via its effects on the noradrenergic system's response to stress [28]. Increasing NPY might be associated with an improvement in anxiety as a cause or result. The cause for the increase in NPY levels with antidepressant treatment may also be up-regulation in the NPY-ergic system being related to antidepressant effect. In animal models of depression, it has been demonstrated that treatments with an antidepressant-like effect increased hippocampal levels of mRNAs encoding NPY and/or the NPY-Y1 receptor, and NPY may stimulate cell proliferation and induce an antidepressant-like response [29]. Previous preclinical and clinical studies suggested that NPY had an antidepressant and anxiolytic-like effect $[4,6]$. Therefore, the NPY-ergic system may be one of the target systems for the development of novel therapeutic agents in the treatment of depression and anxiety. The finding of the present study is consistent with previous studies and might provide evidence for this suggestion.

NPY levels increased after 8 weeks of treatment and increased further after 6 months of treatment. NPY levels were increased by treatment with escitalopram and venlafaxine, but not with sertraline and fluoxetine. We may conclude that the duration of treatment and the type of antidepressant agent used may have a different effect on NPY. Some preclinical studies demonstrated that fluoxetine led to decreased orexigenic neuropeptides such as NPY and anorectic effect. They suggested that a fluoxetine raised level of 5-HT plays an inhibitory role in orectic action $[30,31]$. A few clinical studies investigated the effect of antidepressants on NPY and found controversial results $[19,20]$. Recent studies found no change in CSF NPY concentration with venla-

\begin{tabular}{|c|c|c|c|}
\hline Subgroups & $\begin{array}{l}\text { Pre-treatment } \\
\text { Median(IR) }\end{array}$ & $\begin{array}{l}\text { After } 8 \text { weeks } \\
\text { treatment } \\
\text { Median(IR) }\end{array}$ & Comparisons \\
\hline \multicolumn{4}{|l|}{ Antidepressant: } \\
\hline Sertraline $(n=9)$ & $68(28)$ & $63(30)$ & $Z=0.770 p=0.441$ \\
\hline Escitalopram $(n=9)$ & $72(39.5)$ & $96(49)^{*}$ & $Z=2.073 p=0.038$ \\
\hline Fluoxetine $(n=7)$ & $51(48)$ & $82(63)$ & $Z=1.014 p=0.310$ \\
\hline Venlefaxine $(n=7)$ & $41(30)$ & $66(27)^{*}$ & $Z=2.197 p=0.028$ \\
\hline \multicolumn{4}{|l|}{ Gender: } \\
\hline Women $(n=27)$ & $62(38)$ & $80(41)^{*}$ & $Z=2.427 p=0.015$ \\
\hline Men $(n=5)$ & $51(59.5)$ & $65(32.5)$ & $Z=0.135 p=0.893$ \\
\hline \multicolumn{4}{|l|}{ Disorder: } \\
\hline Major depressive disorder $(n=6)$ & $68.5(57.5)$ & $81(62.25)^{*}$ & $Z=1.992 p=0.046$ \\
\hline Generalized anxiety disorder $(n=5)$ & $67(43.5)$ & $88(68.5)$ & $Z=1.753 p=0.080$ \\
\hline Adjustment disorder with depression $(n=11)$ & $57(32)$ & $77(39)$ & $Z=1.600 p=0.110$ \\
\hline Adjustment disorder with anxiety $(n=10)$ & $65(43.75)$ & $58.5(42.75)$ & $Z=0.102 p=0.919$ \\
\hline
\end{tabular}

IR: Interquartile range

${ }^{*}$ : Higher than those of the pre-treatment 
faxine treatment in humans [14] and no change in hippocampal NPY expression with escitalopram treatment in rats [29]. The increase in serum NPY levels with venlafaxine in the present study may be related to the noradrenergic effect of venlafaxine. Fluoxetine and sertraline seem to be relatively more related to anorectic effect than other SSRIs [32]. The absence of change in NPY levels with fluoxetine and sertraline may be associated with the anorectic effect of these agents, at least in the acute period. Additionally, in the patients diagnosed with major depressive disorder, NPY levels were increased by treatment, but they did not increase in patients with GAD and adjustment disorder. We may interpret this result together with the literature data as the increasing effect of antidepressants on NPY levels may be peculiar to depression. NPY levels were also increased in women, but not in men. However, the small size of subgroups may make assessment difficult.

The major limitation of the present study was that we measured peripheral NPY levels instead of central NPY levels, because the relationship between central and peripheral NPY has not been clarified. Some studies demonstrated no correlation between CSF and plasma NPY levels in non-psychiatric populations, $[33,34]$ although plasma NPY levels are used as a proxy of central NPY activity in some studies [25]. However, peripheral NPY is suggested as a marker of stress-resilience [21]. Low levels of NPY were found in both the CSF and plasma samples of patients with post-traumatic stress disorder $[15,16]$ and also in those patients with depression [8-11]. Therefore, peripheral NPY may be related to psychopathology in stress-related disorders and may partly reflect central NPY signaling [5]. As another limitation of the present study, the patients had high BMI. NPY, which has an orexigenic effect, [26] might be reduced due to regulating weight gain. The low NPY in the patients may be a result of high weight in the present study. However, we tried to neutralize the effect of BMI by taking it as a covariate in the statistical analysis. The result, low NPY in patients, remained the same. Consequently, NPY seems to decrease in patients with no relation to weight gain. As another limitation, our study population did not consist of patients with depression or anxiety alone. Therefore, the heterogeneity of our study population may make assessment difficult. Additionally, the sample size of men was not large enough for interpretation, which is a limitation of the study. The role of gender in the effect of antidepressants may be another interesting issue that is worth investigating in future studies.

\section{Conclusion}

$\nabla$

In conclusion, the study revealed that serum NPY levels were low in patients with depression and anxiety, and they increased and were normalized by antidepressant treatment. The association between antidepressants and NPY levels seems more obvious in depressive patients on certain antidepressants and with long-term treatment. We may speculate that NPY may be a state marker especially for depression. Of course, these suggestions need clarification with further studies.

\section{Acknowledgment}

The study was conducted at Erciyes University and supported by the Research Fund of Erciyes University.

\section{Conflict of Interest}

$\nabla$

The authors declare no conflict of interest.

\section{References}

1 Gehlert DR. Introduction to the reviews on neuropeptide Y. Neuropeptides 2004; 38: 135-140

2 Morales-Medina JC, Dumont Y, Quirion R. A possible role of neuropeptide $Y$ in depression and stress. Brain Res 2010; 1314: 194-205

3 Westfall TC, Martin J, Chen XL et al. Cardiovascular effects and modulation of noradrenergic neurotransmission following central and peripheral administration of neuropeptide Y. Synapse 1988; 2: 299-307

$4 \mathrm{Wu}$ G, Feder A, Wegener $\mathrm{G}$ et al. Central functions of neuropeptide $\mathrm{Y}$ in mood and anxiety disorders. Expert Opin Ther Targets 2011; 15: 1317-1331

5 Heilig $M$. The NPY system in stress, anxiety and depression. Neuropeptides 2004; 38: 213-224

6 Gelfo F, De Bartolo P, Tirassa P et al. Intraperitoneal injection of neuropeptide Y (NPY) alters neurotrophin rat hypothalamic levels: Implications for NPY potential role in stress-related disorders. Peptides 2011; 32: $1320-1323$

7 Antonijevic IA, Murck H, Bohlhalter S et al. Neuropeptide Y promotes sleep and inhibits ACTH and cortisol release in young men. Neuropharmacology 2000; 39: 1474-1481

8 Heilig $M$, Zachrisson 0 , Thorsell A et al. Decreased cerebrospinal fluid neuropeptide Y (NPY) in patients with treatment refractory unipolar major depression: preliminary evidence for association with preproNPY gene polymorphism. J Psychiatry Res 2004; 38: 113-121

9 Hashimoto H, Onishi $H$, Koide S et al. Plasma neuropeptide $Y$ in patients with major depressive disorder. Neurosci Lett 1996; 216: 57-60

10 Hou C, Jia F, Liu Y et al. CSF serotonin, 5-hydroxyindolacetic acid and neuropeptide Y levels in severe major depressive disorder. Brain Res 2006; 1095: 154-158

11 Gulec MY, Ozalmete OA, Ozturk M et al. Plasma neuropeptide Y levels in medication naive adolescents with major depressive disorder. Bulletin of Clinical Psychopharmacology 2010; 20: 132-138

12 Irwin $M$, Brown $M$, Patterson T et al. Neuropeptide $Y$ and natural killer cell activity: findings in depression and Alzheimer caregiver stress. FASEB J 1991; 5: 3100-3107

13 Czermak C, Hauger R, Drevets WC et al. Plasma NPY concentrations during tryptophan and sham depletion in medication-free patients with remitted depression. J Affect Disord 2008; 110: 277-281

14 Martinez JM, Garakani A, Yehuda R et al. Proinflammatory and "resiliency" proteins in the CSF of patients with major depression. Depress Anxiety 2012; 29: 32-38

15 Sah R, Ekhator NN, Jefferson-Wilson L et al. Cerebrospinal fluid neuropeptide $Y$ in combat veterans with and without posttraumatic stress disorder. Psychoneuroendocrinology 2014; 40: 277-283

16 Rasmusson AM, Hauger RL, Morgan CA et al. Low baseline and yohimbine-stimulated plasma neuropeptide Y (NPY) levels in combatrelated PTSD. Biol Psychiatry 2000; 47: 526-539

17 Stein MB, Hauger RL, Dhalla KS et al. Plasma neuropeptide Y in anxiety disorders: findings in panic disorder and social phobia. Psychiatry Res 1996; 59: 183-188

18 Boulenger JP, Jerabek I, Jolicoeur FB et al. Elevated plasma levels of neuropeptide $\mathrm{Y}$ in patients with panic disorder. Am J Psychiatry 1996; 153: $114-116$

19 Nikisch G, Agren H, Eap CB et al. Neuropeptide Y and corticotropinreleasing hormone in CSF mark response to antidepressive treatment with citalopram. Int J Neuropsychopharmacol 2005; 8: 403-410

20 Olsson A, Regnell G, Traskman-Bendz L et al. Cerebrospinal neuropeptide $\mathrm{Y}$ and substance $\mathrm{P}$ in suicide attempters during long-term antidepressant treatment. Eur Neuropsychopharmacol 2004; 14: 479-485

21 Morgan CA 3rd, Wang S, Southwick SM et al. Plasma neuropeptide-Y concentrations in humans exposed to military survival training. Biol Psychiatry 2000; 47: 902-909

22 Doruk A, Çelik C, Özdemir B et al. Adjustment disorder and life events. Anatolian Journal of Psychiatry 2008; 9: 197-202

23 Öznur T. Clinical and differential diagnosis of adjustment disorder. Turkiye Klinikleri J Psychiatry-Special Topics 2015; 8: 53-60

24 Özcan $M$, Uğuz F, Çilli AS. The prevalence of generalized anxiety disorder and comorbidity among psychiatric outpatients. Turkish Journal of Psychiatry 2006; 17: 276-285

25 Sah R, Geracioti TD, Neuropeptide $Y$. and posttraumatic stress disorder. Mol Psychiatry 2013; 18: 646-655

26 Schwartz MW, Woods SC, Porte D Jr et al. Central nervous system control of food intake. Nature 2000; 404: 661-671 
27 Ising M, Kunzel HE, Binder EB et al. The combined dexamethasone/CRH test as a potential surrogate marker in depression. Prog Neuropsychopharmacol Biol Psychiatry 2005; 29: 1085-1093

28 Rasmusson AM, Southwick SM, Hauger RL et al. Plasma neuropeptide $\mathrm{Y}$ (NPY) increases in humans in response to the alpha 2 antagonist yohimbine. Neuropsychopharmacology 1998; 19: 95-98

29 Bjornebekk A, Mathe AA, Brene S. The antidepressant effects of running and escitalopram are associated with levels of hippocampal NPY and Y1 receptor but not cell proliferation in a rat model of depression. Hippocampus 2010; 20: 820-828

30 Myung CS, Kim BT, Choi SH et al. Role of neuropeptide Y and proopiomelanocortin in fluoxetine-induced anorexia. Arch Pharm Res 2005; 28: $716-721$
31 Gutierrez A, Saracibar G, Casis L et al. Effects of fluoxetine administration on neuropeptide y and orexins in obese zucker rat hypothalamus. Obes Res 2002; 10: 532-540

32 Fava $M$, Judge $R$, Hoog SL et al. Fluoxetine versus sertraline and paroxetine in major depressive disorder: changes in weight with long-term treatment. J Clin Psychiatry 2000; 61: 863-867

33 Baker DG, Bertram TM, Patel PM et al. Characterization of cerebrospinal fluid (CSF) and plasma NPY levels in normal volunteers over a 24-h timeframe. Psychoneuroendocrinology 2013; 38: 2378-2382

34 Nam SY, Kratzsch J, Kim KW et al. Cerebrospinal fluid and plasma concentrations of leptin, NPY, and alpha-MSH in obese women and their relationship to negative energy balance. J Clin Endocrinol Metab 2001; 86: 4849-4853 